\section{Extensive central nervous system cryptococcal disease presenting as immune reconstitution syndrome in a patient with advanced HIV: report of a case and review of management dilemmas and strategies}

Onyema Ogbuagu, Merceditas Villanueva

Yale HIV/AIDS Program, Section of

Infectious Diseases, Yale University

School of Medicine, New Haven, CT, USA

\section{Abstract}

One of the complications of the use of antiretroviral therapy (ART), immune reconstitution inflammatory syndrome (IRIS), is particularly problematic in the management of cryptococcal meningitis. We present the case of a 35year-old male with acquired immune deficiency syndrome diagnosed with extensive central nervous system (CNS) cryptococcal disease, including meningitis and multiple intracranial cysts, diagnosed eight weeks after the initiation of ART. The patient experienced a relapsing and remitting clinical course despite repeated courses of potent antifungal therapy and aggressive management of raised intracranial pressure. This review highlights therapeutic dilemmas and strategies in the management of CNS cryptococcosis complicated with IRIS and highlights gaps in available treatment guidelines.

\section{Introduction}

Despite the widespread availability and use of antiretroviral therapy (ART), opportunistic infections continue to cause significant morbidity and mortality among individuals infected with human immunodeficiency virus (HIV) ${ }^{1-3}$ One of the adverse consequences of exposure to ART is the development of immune reconstitution inflammatory syndrome (IRIS) which may manifest as a newly identified opportunistic infection (OI) in previously asymptomatic individuals (unmasking IRIS) or with paradoxical clinical worsening of a known OI (paradoxical IRIS). ${ }^{4-6}$ Patients who develop IRIS may experience prolonged or increased rates of hospitalization, disability and increased mortality. ${ }^{7}$ IRIS involving OI's of the central nervous system (CNS) may be particularly life-threatening. ${ }^{8-10}$

Central nervous system cryptococcal disease is a common OI that can present as IRIS. It may mimic disease relapse and, therefore, can be puzzling to providers managing the condition. ${ }^{11-13}$ This case-based review will describe a challenging presentation of CNS cryptococcal IRIS disease in a patient with acquired immune deficiency syndrome (AIDS) and highlights dilemmas in the management of the disease that are not fully addressed by available treatment guidelines.

\section{Case Report}

A 35-year-old male with an 8-year history of AIDS presented with a 3-day history of recurrent frontal headaches, subjective fever and altered mental status. He had a history of non-adherence to medications, but he had resumed antiretroviral drugs for about 10 weeks. Four weeks prior to presentation to our hospital, he had been diagnosed with cryptococcal meningitis (CM) in an outside hospital and had received a 7 day course of intravenous amphotericin B (lipid complex preparation). This was discontinued due to progressive acute kidney injury and he was subsequently placed on high dose ( 800 $\mathrm{mg}$ ) oral fluconazole (FLU) daily.

At the antecedent hospital visit, his presenting CD4 count was 16 cells/ $\mu \mathrm{L}$ and plasma HIV RNA viral load was 1970 copies $/ \mathrm{mL}$, a decrease from a viral load of 50,000 copies/mL at treatment re-initiation 8 weeks previously. His viral load had further declined to less than 20 copies/mL at the time of initial evaluation at our facility 4 weeks later. His nadir CD4 count was 9 cells/ $\mu \mathrm{L}$ (Table 1). His antiretroviral drug regimen consisted of darunavir boosted with ritonavir, tenofovir and emtricitabine taken once daily. He reported at least 2 prior episodes of $\mathrm{CM}$ with the most recent and preceding episode occurring about 3 years ago. He had not been on antifungal prophylaxis prior to his current illness.

On presentation, he was cachectic, but was in no acute distress. Temperature was $36.9^{\circ} \mathrm{C}$, heart rate 84 beats/minute, $\mathrm{BP} 87 / 65 \mathrm{mmHg}$, respiratory rate 18 cycles/minute and oxygen saturation of $100 \%$ on room air. He was alert and oriented to time, place and person; a mini mental status examination score was 25 ; he exhibited photophobia but no other meningeal signs. He had bilateral horizontal nystagmus and mild right upper extremity hemiparesis. Cardio-pulmonary exam was unremarkable.

Peripheral white blood cell (WBC) count was 2900 cells/ $\mu \mathrm{L}$ with a normal differential. Although no formal measurements were taken, a spinal tap was consistent with elevated opening pressure as there was brisk cerebrospinal fluid (CSF) flow. CSF analysis showed a WBC count of 41 cells per high power field comprising $75 \%$ lymphocytes, protein - $800 \mathrm{mg} / \mathrm{dL}(<50$ $\mathrm{mg} / \mathrm{dL}$ - normal), and glucose level of $13 \mathrm{mg} / \mathrm{dL}$
Correspondence: Onyema Ogbuagu, Yale HIV/AIDS Program, Section of Infectious Diseases, Yale University School of Medicine, 135 College Street, Suite 323, New Haven, CT 06519, USA.

Tel.: +1.516.281.4557 - Fax: +1.203.737.4051

E-mail: onyema.ogbuagu@yale.edu

Key words: cryptococcal meningitis, immune reconstitution inflammatory syndrome, acquired immune deficiency syndrome.

Contributions: 00, conception of the article, drafting, editing and reviewing the manuscript; $\mathrm{MV}$, drafting, editing and reviewing the manuscript.

Conflict of interests: the authors declare no potential conflict of interests.

Received for publication: 28 July 2014

Revision received: 13 August 2014

Accepted for publication: 14 August 2014.

This work is licensed under a Creative Commons Attribution NonCommercial 3.0 License (CC BYNC 3.0).

(OC) Copright O. Ogbuagu and M. Villanueva, 2014 Licensee PAGEPress, Italy

Infectious Disease Reports 2014; 6:5576 doi:10.4081/idr.2014.5576

(40-70 mg/dL - normal). CSF India Ink stain demonstrated yeast but there was no subsequent growth on culture after 4 weeks of incubation. CSF and serum cryptococcal antigen (CRAG) titers were $>1: 1024$ and 1:512 respectively. Blood cultures showed no growth.

A brain magnetic resonance image (MRI) with gadolinium showed clusters of gelatinous cystic lesions along the periventricular white matter structures and within the lateral ventricles, basal ganglia and posterior cranial fossa. There was enlargement of the ventricular system with no evidence of structural obstruction to CSF flow to suggest communicating hydrocephalus. It also showed diffuse leptomeningeal and ependymal enhancement consistent with meningitis and ventriculitis (Figure 1).The patient was started on induction therapy with liposomal amphotericin B (LAmB) $6 \mathrm{mg} / \mathrm{kg} / \mathrm{d}$ and flucytosine (5FC) 100 $\mathrm{mg} / \mathrm{kg} / \mathrm{d}$ for 2 weeks and subsequently transitioned to $400 \mathrm{mg}$ oral FLU with resolution of motor deficits and improved CSF parameters (Table 1). His ART was continued and he was discharged home.

He was readmitted to the hospital 5 weeks later with recurrent headaches and fevers. MRI of his brain showed no change. Repeat CSF evaluation showed persistent lymphocytic pleocytosis, hypoglycorrhachia and an elevated opening pressure of $38 \mathrm{~cm} \mathrm{H}_{2} \mathrm{O}$. There were no cryptococcal organisms noted on fungal stain 
and culture eventually showed no growth. At this point, it was suspected that his presentation was at least in part due to IRIS. However, because of concern for his persistent cerebral mass lesions, ventriculitis and CSF abnormalities, it was decided to treat him with a prolonged induction course ( 8 weeks) of LAmB and 5FC followed by FLU consolidation therapy (400 mg daily oral dose). He underwent serial LPs during this course which showed persistently elevated protein, low glucose and high CRAG titers (weeks 12-20 in Table 1).

Four months later, he required placement of a ventriculoperitoneal shunt for persistently elevated CSF pressures. A biopsy of one of his CNS cysts at the time showed an aggregation of organisms morphologically consistent with Cryptococcus species on pathologic examination. No cultures were performed. He was maintained on oral FLU $200 \mathrm{mg}$ daily. On outpatient follow up 1 year later, he demonstrated severe cognitive impairment. Our patient was subsequently lost to follow up and 2 years after his initial presentation, he died. Proximate cause of death remains unknown.

\section{Discussion}

Cryptococcal meningitis remains a significant cause of morbidity and mortality among patients with AIDS. In recent years, the clinical outcomes of AIDS patients with cryptococcal disease has improved due to the increased availability and use of antiretroviral drugs, adoption of more potent formulations and combinations of antifungal agents, and recognition of the importance of aggressive management of raised intracranial pressure..$^{14,15}$ Nonetheless, the mortality remains high, ranging from 5.5 to $25 \% .{ }^{16,17}$

Cryptococcus neoformans exists in nature predominantly as an encapsulated budding yeast and has a worldwide distribution. ${ }^{18,19}$ The organism is found in vegetative matter such as tree barks as well as in bird (pigeon) droppings. Human infection is acquired via inhalation of infectious spores resulting initially in pulmonary disease. Hematogenous dissemination is thought to be the principal method of extra-pulmonary organ involvement. ${ }^{18}$ The predominant species which cause human disease are Cryptococcus neoformans var neoformans, Cryptococcus neoformans var grubii and Cryptococcus gattii. ${ }^{20,21}$

Cryptococcus gattii is an increasingly recognized cause of more severe cryptococcal disease in both immunocompetent and immunodeficient individuals. This species has a predilection for the CNS including formation of cryptococcomas, and demonstrates a poorer response to antifungal therapy. ${ }^{19,22}$ In clinical practice, speciation of cultured cryptococcal isolates is not routinely performed and the management of infection by both species is the same. ${ }^{16}$

Cryptococcal disease in HIV-infected individuals most commonly involves the CNS. Occasionally, it may manifest as isolated pulmonary disease, cutaneous lesions or may be disseminated. HIV-infected individuals are more likely to have CNS disease compared to immunocompetent individuals or those with other acquired immunodeficiency states such as solid organ transplant recipients. ${ }^{23,24}$

Central nervous system disease generally manifests as a meningoencephalitis and/or mass lesions. Four distinct patterns of CNS mass lesions have been described: cryptococcomas, cystic lesions alone, mixed cysts and cryptococcomas, and miliary nodules. ${ }^{25,26}$ CNS cryptococcomas are radiologically defined as intra-parenchymal lesions with or without enhancement on computerized tomography (CT) imaging and histologically, they represent a chronic granulomatous reaction to fungal invasion. ${ }^{26}$ However, this immune response may be diminished in severely immunosuppressed individuals who are less likely to present with cryptococcomas.

Cryptococcal cysts have been reported in non-HIV and HIV-infected individuals, ${ }^{27,28}$ but the actual prevalence is not known. In an autopsy study, gelatinous pseudocysts were noted in 30 out of 45 AIDS patients who died of CNS cryptococcosis. ${ }^{29}$ These cystic structures appear to be morphologically unique entities and are distinct from solid cryptococcomas on imaging and by pathologic examination. . $^{30,31}$ They most likely represent dilated VirchowRobin (peri-vascular) spaces filled with fungi and sequestered cerebrospinal fluid and the usual sites where these have been identified include the basal ganglia and midbrain. ${ }^{32}$ The cysts may be so large to cause pressure-related complications. Large gelatinous pseudocysts causing brain stem compression with seizures in an 11 year old immunocompetent female were described in Indiana. ${ }^{33}$ In some instances, the cysts have been reported to persist despite the successful treatment of the underlying disease. ${ }^{30}$

On brain imaging, our patient was found to have cystic lesions in the periventricular white matter, in both lateral ventricles and basal ganglia. These are classic locations for cryptococcal mass lesions which are best detected by magnetic resonance imaging (MRI). A single center retrospective review of 29 patients with CNS cryptococcal disease, the majority of whom were HIV positive, evaluated the performance of different modalities of brain imaging: computerized tomography (CT) versus MRI in detection of lesions associated with intracranial cryptococcosis. ${ }^{32}$ The study found that MRI was more sensitive than CT scan in detecting abnormalities; notably, half of the patients with abnormal MRI scans had normal CT scans. Three patients who subsequently died were found with cystic lesions in their basal ganglia on autopsy that corresponded with their MRI findings.

Our patient demonstrated clinical signs and symptoms of cryptococcal meningitis at his initial presentation which was 8 weeks after he reinitiated ART. Although he had previously been diagnosed with $\mathrm{CM}$, he had been out of care and presumably asymptomatic for a number of years. The development of recurrent symptoms of CNS cryptococcosis in the setting of an initial low CD4 count with subsequent decline in HIV viral load on antiretroviral therapy is compatible with unmasking IRIS. During his subsequent presentations to care, the absence of growth of cryptococcal organisms in CSF culture also supports IRIS as the etiology of his recurrent symptoms rather than progressive disease. We also believe that the large burden of cryptococcal organisms in the setting of aggregated cysts may have contributed to his clinical relapses as evidenced by his persistent CSF and radiologic abnormalities.

We faced the following dilemmas in the management of our patient.

\section{Management of antiretroviral therapy in central nervous system cryptococcal immune reconstitution inflammatory syndrome}

The appropriate timing of initiation of ART in HIV infected patients with CM used to be controversial. However, based on 2 recent clinical trials, it is now accepted that early initiation of HAART in antiretroviral treatment naïve patients with CM results in excess mortality that is at least in part due to paradoxical IRIS. ${ }^{34-36}$ On the other hand, a meta-analysis of 2 published randomized controlled trials showed no statistically significant difference in mortality between CM patients with early ( $<4$ weeks) versus delayed initiation of ART ( $>4$ weeks). ${ }^{37}$ One theoretical advantage to the early use of ART in patients with cryptococcal meningitis is that the associated immune reconstitution should improve clearance of the fungal organisms. However, this has not been observed in clinical practice. A prospective study conducted among ART-naïve patients in Botswana showed no benefit of early initiation of ART (within 7 days) versus delayed therapy (after 28 days) on CSF clearance of cryptococcal organisms despite high rates of IRIS in the early ART group. ${ }^{38}$

There is no data on approaches to the use of ART in patients with unmasking cryptococcal IRIS, such as in our patient. Is there a role for 
temporary treatment discontinuation? It is certainly plausible that continuation of ART in individuals with CNS cryptococcal IRIS may be similarly associated with increased mortality and morbidity as in individuals who are first initiated on ART early after the event. The alternative, treatment interruption, may have risks including antiretroviral drug resistance as well as AIDS progression and increased mortality. ${ }^{39-41}$

We elected to continue our patient's ART regimen while aggressively controlling his raised intracranial pressure which is a principal cause of morbidity and mortality in CNS CM with or without IRIS. Finally, to date, studies assessing the impact of the timing of introduction of ART among HIV infected patients with CM have focused primarily on mortality outcomes. However, important morbidity outcomes such as neurocognitive and visual complications have largely been ignored and need to be evaluated in high quality studies. ${ }^{42}$

\section{Distinguishing central nervous system cryptococcal immune reconstitution inflammatory syndrome from persistent or progressive disease}

Distinguishing between progressive disease and IRIS in patients with CNS cryptococcosis can be challenging but is crucial for determining appropriate management. ${ }^{43}$ In the first scenario, the primary focus of management would include a consideration of resumption of and/or prolonged antifungal induction therapy to decrease fungal burden and sterilize infected sites. In the latter case, the goal would be to address inflammatory complications including elevated CSF pressures. Clinically, a worsening of clinical symptoms after an initial improve- ment, despite potent antifungal therapy and in the setting of ART-associated HIV disease control, favors IRIS..$^{44}$ Otherwise, the clinical presentation of IRIS is indistinguishable from progressive cryptococcal disease. Our patient experienced a relapsing and remitting course of disease. Serial lumbar punctures and CSF analysis continued to show significant derangements including elevated opening pressure, elevated protein, hypoglycorrhachia and lymphocytic pleocytosis concerning for persistent cryptococcal disease (Table 1). Cryptococcal antigen titers remained high at 1:512 or greater. Brain imaging showed persistence of his CNS cystic lesions (Figure 1).

The use of laboratory tests to distinguish between cryptococcal IRIS and persistent disease can be challenging. A study by Boulware and colleagues compared CSF parameters at baseline and at the time of development of IRIS in patients with HIV infection and CM. During IRIS compared to baseline, fungal cultures were more likely to be negative (median of 0 versus 13.3 colony forming units $/ \mathrm{mL}$ on culture, $\mathrm{P}<0.001$ ) and CSF cryptococcal antigen titers were lower (median of 1:1024 versus $1: 32, \mathrm{P}<0.001){ }^{45}$ On the other hand, median CSF protein and leukocytosis were significantly increased in patients with IRIS as were median levels of inflammatory cytokineseotaxin, tumor necrosis factor alpha (TNF $\alpha)$, interferon gamma (IFN $\gamma$ ), granulocyte colony stimulating factor (G-CSF) and vascularendothelial growth factor (VEGF). The subgroup of patients in the study with CM relapse had lower levels of TNF $\alpha$, IFN $\gamma$, interleukins (IL) 4,9,12 and 17 compared to those who developed IRIS suggesting a more intense

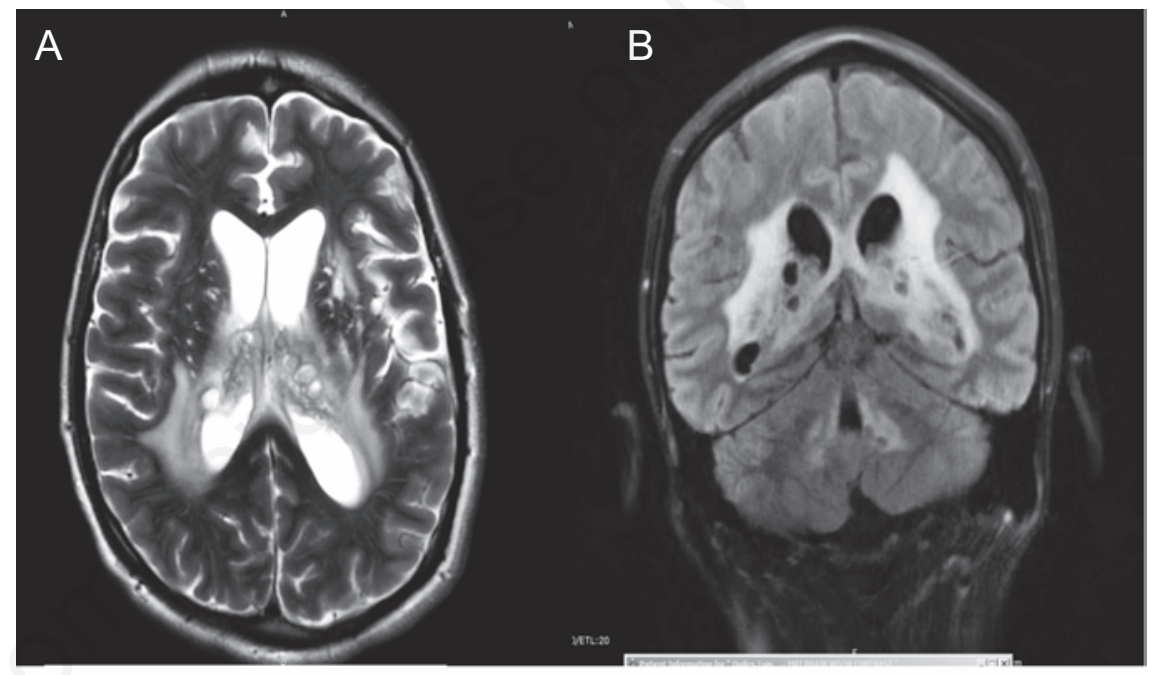

Figure 1. Brain magnetic resonance image: axial and coronal views showing clusters of cystic structures within the lateral ventricles periventricular white matter area and basal ganglia. There is enlargement of the ventricular system as well. B) shows additional cystic lesions surrounding the fourth ventricle.

Table 1. Trend of CD4 count and HIV viral load 8 weeks prior to initial presentation, as well as cerebrospinal fluid parameters and antifungal induction treatment courses of the index patient during the first 5 months of his presentation.

\begin{tabular}{|c|c|c|c|c|c|c|c|}
\hline & Week -8 & Week 1* & Week 5 & Week 12 & Week 14 & Week 16 & Week 20 \\
\hline CSF CRAG & - & $1: 512$ & $>1: 1024$ & $>1: 1024$ & $>1: 1024$ & $1: 512$ & $>1: 1024$ \\
\hline CSF opening pressure & - & Brisk flow & NR & 38 & NR & NR & 35 \\
\hline CSF WBC (\% lymphs) & - & $24(84 \%)$ & $41(75 \%)$ & $12(64 \%)$ & $6(55 \%)$ & $14(92 \%)$ & $23(56 \%)$ \\
\hline CSF protein & - & 702 & 800 & 860 & 650 & 256 & 542 \\
\hline CSF glucose & - & 6 & 13 & 17 & 24 & 33 & 15 \\
\hline CSF fungal stain & - & Negative & $1+$ yeast & Negative & Negative & $1+$ yeast & Negative \\
\hline CSF culture & - & C. neoformans & No growth & No growth & No growth & No growth & No growth \\
\hline HIV VL (copies/mL) & 50,000 & 1740 & Not detected & $<20$ & & & \\
\hline 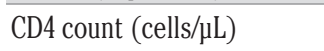 & 9 (nadir) & 16 & 16 & 29 & & & \\
\hline Antifungal induction therapy & & AmB (1 week) & LAmB/5-FC (2 weeks) & LAmB/5-FC (8 weeks) & & & \\
\hline
\end{tabular}


inflammatory response in the latter group. Limiting the utility of these observations is that no cut-off values for the CSF cytokines have been established to distinguish the two clinical entities. In our patient, the CSF India ink and cryptococcal cultures were negative at time of subsequent presentations favoring IRIS but he continued to show elevated CRAG levels, persistent radiologic and CSF abnormalities concerning for persistent disease. Obtaining additional inflammatory markers such as CSF cytokine levels was not feasible.

\section{Duration of antifungal induc- tion therapy in central nervous system cryptococcal disease with mass lesions}

The optimal duration of treatment of CNS cryptococcosis in the setting of cryptococcomas or cystic lesions in HIV infected individuals is not well studied. The Infectious Diseases Society of America (IDSA) guidelines for treatment of cryptococcomas suggests an antifungal induction period of at least 6 weeks; this is based primarily on expert opinion and case reports, as well as observational and retrospective studies mostly in non HIV-infected individuals. ${ }^{16,46}$ It is not clear that these recommendations extend to treatment of cryptococcal cysts which are distinct pathologic entities that may have differing responses to antifungal therapy.

Histopathologic examination shows that cryptococcal cysts consist of gelatinous capsular aggregations of fungal organisms which represent protected foci potentially with increased resistance to antifungal therapy due to protective biofilm. ${ }^{47}$ These clusters of organisms may remain viable despite long-term antifungal therapy and may be the cause of recrudescence when therapy is discontinued.

The prominent cryptococcal polysaccharide capsule is a well-known means by which the fungus evades immunologic clearance. ${ }^{48}$ Immune system evasion is also enhanced by quorum sensing mechanisms when cryptococcal organisms aggregate to form biofilm. ${ }^{49} \mathrm{~A}$ study by Cordoba and colleagues explored the impact of cryptococcal organism capsule size on time-kill curves following exposure to amphotericin B (AmB).$^{50}$ Large capsule organisms $(10-15 \mu \mathrm{m})$ compared to small capsule (2$4 \mu \mathrm{m})$ organisms showed more resistance to AmB killing over time. When exposed to 1 microgram $/ \mathrm{mL}$ concentration of $\mathrm{AmB}$, at 24 hours of incubation, $95 \%$ of large capsule isolates survived compared to $15 \%$ of small capsule isolates. Furthermore, in vitro studies show that cryptococcal organisms organized in biofilms, compared to planktonic forms, show increased resistance to $\mathrm{AmB}$ and complete resistance to azole activity. ${ }^{51}$ More studies are needed to explore the optimal management of CNS cryptococcal mass lesions. Our patient continued to have persistent abnormal CSF parameters despite a second course of prolonged antifungal induction therapy perhaps due to his extensive CNS mass lesions. Because cryptococcal cultures yielded no further growth, resistance testing was unable to be performed. However, resistance to $\mathrm{AmB}$ and $5 \mathrm{FC}$ by wild type cryptococcal isolates is rare. ${ }^{52}$

\section{Role of intrathecal antifungals}

The poor CNS penetration of intravenously (IV) administered AmB makes intrathecal administration an attractive option. ${ }^{53,54} \mathrm{~A}$ retrospective review of $2068 \mathrm{CM}$ cases in China, with HIV-infected individuals comprising 2-5\% of study subjects, assessed the impact of the addition of intrathecal $\mathrm{AmB}$ (iAmB) to each of four treatment groups: systemic $\mathrm{AmB}, \mathrm{AmB}+5 \mathrm{FC}$, $\mathrm{AmB}+\mathrm{FLU}$ and $\mathrm{AmB}+5 \mathrm{FC}+\mathrm{FLU}$ on treatment outcomes. $^{55}$ Mortality rate was significantly lower in the iAmB arms compared to the noniAmB control arm in the first three treatment groups ( $6 \%$ vs $23 \%$, $25 \%$ vs $35 \%$, $20 \%$ vs $30 \%$, respectively, $\mathrm{P}<0.05)$. There were also significant clinical improvement and cure rates in the same groups as well. The addition of iAmB did not impact mortality in the $\mathrm{AmB}+5 \mathrm{FC}+\mathrm{FLU}$ arm. However, the use of ventricular drainage (through serial lumbar punctures and ventricular shunts), well known to improve mortality outcomes, were higher overall in patients treated with iAmB across almost all treatment groups (except the AmB + 5FC group) and may have favorably influenced results.

A treatment protocol reported by Yao et al., in a therapeutic trial of $\mathrm{iAmB}$ for $\mathrm{CM}$ in immunocompetent patients with the objective of improving cure rates, involved the administration of iAmB at a daily dose ranging from 0.1 to $1.0 \mathrm{mg} / \mathrm{d}$ dose given twice a week and continued until the CSF cryptococcal cultures were negative. The intrathecal injection consisted of a $1.0 \mathrm{mg} / \mathrm{mL}$ concentration of AmB reconstituted with injection water, admixed with 4-5 $\mathrm{mL}$ of retrieved $\mathrm{CSF}$ and given slowly; coadministered with 1-2 $\mathrm{mg}$ of dexamethasone over the treatment period. ${ }^{56}$ This was tried in 10 patients who did not have underlying immunodeficiency states; 1 patient developed urinary retention after 25 doses of treatment. All were reported to have been successfully cured with no disease recurrence.

The use of iAmB is not without complications. In a small study describing the successful treatment of cryptococcal meningitis in 14 HIV-negative patients with a unique protocol of 8 weeks of antifungal induction therapy using IV or intrathecal AmB (if intolerant of IV form) and 5FC with lumbar drainage, a 50\% complication rate was observed. The predominant adverse effects were lumbosacral nerve irritation and urinary retention. The latter side effect was generally mild and resolved with AmB dose reduction. ${ }^{57}$ No infections related to therapeutic lumbar procedures were reported among study participants.

The routine use of intrathecal antifungals is not currently recommended due to insufficient data in HIV-infected individuals, its prohibitive cost and concern for side effects of therapy. Additionally, multiple studies have shown favorable clinical results with conventional IV and oral antifungal therapy. It remains to be seen if certain subgroups of patients would potentially benefit from such aggressive medical therapy.

\section{Role of steroids in severe central nervous system crypto- coccosis}

While the role of systemic steroids has been better elucidated in certain HIV associated opportunistic infections like tuberculous meningitis, its utility in CNS cryptococcosis is less clear. There are no randomized controlled trials studying the risks and benefits of steroid use in severe CNS cryptococcal disease or IRIS. However, there are multiple case reports of therapeutic success and good neurologic outcomes with the use of adjunctive steroids in HIV and non HIV- infected individuals with severe CNS cryptococcosis, ${ }^{58-61}$ but these reports likely reflect reporting bias for positive treatment outcomes.

Improvement in visual outcomes has been reported with adjunctive steroid use. ${ }^{62,63}$ However, since steroid use is typically performed in conjunction with other interventions including the control of intracranial pressure and antifungal therapy, it is difficult to assess the independent beneficial role of steroids. Furthermore, not all studies have shown a therapeutic benefit. One retrospective case-control analysis of patients with visual loss and cryptococcal meningitis study who did or did not receive corticosteroids showed no benefit to steroid use for visual outcomes although the sample size was small. ${ }^{64}$ Because of the lack of high quality data supportive of the benefits of steroid use, it is not routinely recommended.

\section{End-point of treatment}

For CNS cryptococcomas, the IDSA suggests a minimum of a 6 week course of antifungal 
induction treatment followed by consolidation and maintenance therapy with high dose FLU (400-800 mg oral daily dose) for a period ranging from 6-18 months based on clinical, mycologic and imaging responses. ${ }^{16}$ In HIV-infected individuals, the IDSA also recommends discontinuation of maintenance therapy if the CD4 count remains above 100 cells/ $\mu \mathrm{L}$ for 3 months and HIV viral load is fully or close to fully suppressed.

Application of these guidelines to a patient such as ours is challenging given that he had a relapsing and remitting clinical course with extensive CNS cerebral cryptococcosis and heavy fungal burden. The use of clinical, mycologic and radiologic responses to guide antifungal treatment discontinuation is not always straightforward. In some cases, clinical resolution may only be temporary and residual neurologic deficits are not uncommon even if immunologic recovery is attained. Clinical disease relapse may occur in the setting of suboptimal therapy, poor medication adherence, as well as confounded by the development of IRIS. Additionally, demonstration of microbiologic clearance of cerebrospinal fluid while a reasonable goal in meningitis, may not be a reliable end-point in patients with solid parenchymal lesions or cysts. Obtaining surgical biopsies to demonstrate sterilization of mass lesions is not practical in most settings. Finally, CNS lesions may persist despite clinical disease eradication, ${ }^{58}$ making complete radiologic resolution of disease an unreliable endpoint.

We endorse the IDSA recommendation that a combination of improved clinical symptoms, microbiologic cure and improvement, if not resolution, of imaging findings should guide duration of treatment rather than rigid pre-set courses of therapy especially in complicated CNS disease.

\section{The role for surgery in cryptococcal central nervous system parenchymal disease}

In cases where lesions are large with severe neurologic symptoms due to compression of intracranial structures or refractory to medical management, surgical excision may be indicated. ${ }^{59}$ Ventricular shunt placement is indicated in some cases for persistently high CSF pressures.

There are rare case reports of successful surgical management of CNS cryptococcomas. ${ }^{65,66}$ However, given the typical locations in the CNS and propensity for multiple lesions, complete surgical resection is usually impossible without the risk of serious neurologic damage. Surgery is therefore, unlikely to play a major role in the management of the majority of cases of CNS cryptococcal disease.

\section{Conclusions}

Our patient presented with extensive CNS cryptococcal disease following ART exposure and experienced paradoxical clinical relapses predominantly due to IRIS. This is supported by his CD4 recovery in the setting of declining and eventually fully suppressed HIV viral load on ART and the negative CSF cultures when he experienced clinical disease flares. He was treated with prolonged courses of antifungal induction therapy and ART was continued despite the evidence for IRIS as is the current standard of care. His extensive CNS fungal burden in the presence of cryptococcal cysts may have impacted his inability to normalize his CSF profile.

Prompt recognition of the various modes of presentation of opportunistic diseases in patients with HIV-related advanced immunodeficiency syndromes and improved management strategies are important to improved patient outcomes. Furthermore, our case highlights that treatments must be individualized and tailored to address unique individual presentations, particularly when published guidelines fall short. We have also highlighted gaps in current knowledge on the management of CNS cryptococcal disease for which better research-based evidence is needed.

\section{References}

1. Modi S, Chiu A, Ng'eno B, et al. Understanding the contribution of common childhood illnesses and opportunistic infections to morbidity and mortality in children living with HIV in resource-limited settings. AIDS 2013;27:S159-67.

2. Lawn SD, Harries AD, Wood R. Strategies to reduce early morbidity and mortality in adults receiving antiretroviral therapy in resource-limited settings. Curr Opin HIV AIDS 2010;5:18-26.

3. Lawn SD, Harries AD, Anglaret X, et al. Early mortality among adults accessing antiretroviral treatment programmes in sub-Saharan Africa. AIDS 2008;22:1897908.

4 Malik A, Khan PA, Shujatullah F, et al. Rapid development of IRIS in the form of cryptococcal meningitis after beginning ART. Med Mycol Case Rep 2012;1:56-8.

5. Ruiz-Cruz M, Alvarado-de la Barrera C, Ablanedo-Terrazas Y, Reyes-Teran G. Proposed clinical case definition for cytomegalovirus-immune recovery retini tis. Clin Infect Dis 2014;59:298-303.

6. Achenbach CJ, Harrington RD, Dhanireddy S, et al. Paradoxical immune reconstitution inflammatory syndrome in HIV-infected patients treated with combination antiretroviral therapy after AIDSdefining opportunistic infection. Clin Infect Dis 2012;54:424-33.

7. Hoyo-Ulloa I, Belaunzarán-Zamudio PF, Crabtree-Ramirez B, et al. Impact of the immune reconstitution inflammatory syndrome (IRIS) on mortality and morbidity in HIV-infected patients in Mexico. Int $\mathrm{J}$ Infect Dis 2011;15:e408-e14.

8. Kiggundu R, Rhein J, Meya DB, et al. Unmasking cryptococcal meningitis immune reconstitution inflammatory syndrome in pregnancy induced by HIV antiretroviral therapy with postpartum paradoxical exacerbation. Med Mycol Case Rep 2014;5:16-9.

9. Guevara-Silva EA, Ramirez-Crescencio MA, Soto-Hernandez JL, Cardenas G. Central nervous system immune reconstitution inflammatory syndrome in AIDS: experience of a Mexican neurological centre. Clin Neurol Neurosurg 2012;114:85261.

10. Bahr N, Boulware DR, Marais S, et al. Central nervous system immune reconstitution inflammatory syndrome. Curr Infect Dis Rep 2013;15:583-93.

11. Wingfield T, Baxter J, Herwadkar A, et al. Persistent cryptococcal brain infection despite prolonged immunorecovery in an HIV-positive patient. Case Rep Neurol Med 2014;2014:164826.

12. Musubire AK, Boulware DR, Meya DB, Rhein J. Diagnosis and management of cryptococcal relapse. J AIDS Clin Res 2013;3:S3-003.

13. Musubire AK, Meya BD, Mayanja-Kizza H, et al. Challenges in diagnosis and management of Cryptococcal immune reconstitution inflammatory syndrome (IRIS) in resource limited settings. Afr Health Sci 2012;12:226-30.

14. Warkentien T, Crum-Cianflone NF. An update on Cryptococcus among HIV-infected patients. Int J STD AIDS 2010;21:67984.

15. Macsween KF, Bicanic T, Brouwer AE, et al. Lumbar drainage for control of raised cerebrospinal fluid pressure in cryptococcal meningitis: case report and review. J Infect 2005;51:e221-4.

16. van der Horst CM, Saag MS, Cloud GA, et al. Treatment of cryptococcal meningitis associated with the acquired immunodeficiency syndrome. National Institute of Allergy and Infectious Diseases Mycoses Study Group and AIDS Clinical Trials Group. N Engl J Med 1997;337:15-21.

17. Bicanic T, Wood R, Meintjes G, et al. High- 
dose amphotericin B with flucytosine for the treatment of cryptococcal meningitis in HIV-infected patients: a randomized trial. Clin Infect Dis 2008;47:123-30.

18. Kozubowski L, Heitman J. Profiling a killer, the development of Cryptococcus neoformans. FEMS Microbiol Rev 2012;36:78-94.

19. Chaturvedi V, Chaturvedi S. Cryptococcus gattii: a resurgent fungal pathogen. Trends Microbiol 2011;19:564-71.

20. Bicanic T, Harrison TS. Cryptococcal meningitis. Br Med Bull 2004;72:99-118.

21. Sanchini A, Smith IM, Sedlacek L, et al. Molecular typing of clinical Cryptococcus neoformans isolates collected in Germany from 2004 to 2010. Med Microbiol Immunol 2014;203:333-40.

22. Byrnes EJ 3rd, Li W, Lewit Y, et al. First reported case of Cryptococcus gattii in the Southeastern USA: implications for travelassociated acquisition of an emerging pathogen. PLoS One 2009;4:e5851.

23. Davis JA, Horn DL, Marr KA, Fishman JA. Central nervous system involvement in cryptococcal infection in individuals after solid organ transplantation or with AIDS Transpl Infect Dis 2009;11:432-7.

24. Lin TY, Yeh KM, Lin JC, et al. Cryptococcal disease in patients with or without human immunodeficiency virus: clinical presentation and monitoring of serum cryptococcal antigen titers. J Microbiol Immunol Infect 2009;42:220-6.

25. Ruiz A, Post MJ, Bundschu CC. Dentate nuclei involvement in AIDS patients with CNS cryptococcosis: imaging findings with pathologic correlation. J Comput Assist Tomogr 1997;21:175-82.

26. Troncoso A, Fumagalli J, Shinzato R, et al. CNS cryptococcoma in an HIV-positive patient. J Int Assoc Physicians AIDS Care (Chic) 2002;1:131-3.

27. Kumari R, Raval M, Dhun A. Cryptococcal choroid plexitis: rare imaging findings of central nervous system cryptococcal infection in an immunocompetent individual. Br J Radiol 2010;83:e14-7.

28. Kovoor JM, Mahadevan A, Narayan JP, et al. Cryptococcal choroid plexitis as a mass lesion: MR imaging and histopathologic correlation. Am J Neuroradiol 2002;23:273 6.

29. Klock C, Cerski M, Goldani LZ. Histopathological aspects of neurocryptococcosis in HIV-infected patients: autopsy report of 45 patients. Int J Surg Pathol 2009;17:444-8.

30. Hospenthal DR, Bennett JE. Persistence of cryptococcomas on neuroimaging. Clin Infect Dis 2000;31:1303-6.

31. Andreula CF, Burdi N, Carella A. CNS cryptococcosis in AIDS: spectrum of MR findings. J Comput Assist Tomogr 1993;17:438
41.

32. Tien RD, Chu PK, Hesselink JR, et al. Intracranial cryptococcosis in immunocompromised patients: CT and MR findings in 29 cases. Am $\mathrm{J}$ Neuroradiol 1991;12:283-9.

33. Caldemeyer KS, Mathews VP, EdwardsBrown MK, Smith RR. Central nervous system cryptococcosis: parenchymal calcification and large gelatinous pseudocysts. Am J Neuroradiol 1997;18:107-9.

34. Boulware DR, Meya DB, Muzoora C, et al. Timing of antiretroviral therapy after diagnosis of cryptococcal meningitis. $\mathrm{N}$ Engl $\mathrm{J}$ Med 2014;370:2487-98.

35. Makadzange AT, Ndhlovu CE, Takarinda K, et al. Early versus delayed initiation of antiretroviral therapy for concurrent HIV infection and cryptococcal meningitis in sub-saharan Africa. Clin Infect Dis 2010;50:1532-8.

36. Jarvis JN, Bicanic T, Loyse A, et al. Determinants of mortality in a combined cohort of 501 patients with HIV-associated Cryptococcal meningitis: implications for improving outcomes. Clin Infect Dis 2014;58:736-45.

37. Njei B, Kongnyuy EJ, Kumar S, et al. Optimal timing for antiretroviral therapy initiation in patients with HIV infection and concurrent cryptococcal meningitis. Cochrane Database Syst Rev 2013;2: Cd009012.

38. Bisson GP, Molefi M, Bellamy S, et al. Early versus delayed antiretroviral therapy and cerebrospinal fluid fungal clearance in adults with HIV and cryptococcal meningitis. Clin Infect Dis 2013;56:1165-73.

39. Zolopa A, Andersen J, Powderly W, et al. Early antiretroviral therapy reduces AIDS progression/death in individuals with acute opportunistic infections: a multicenter randomized strategy trial. PLoS One 2009;4:e5575.

40. Holkmann Olsen C, Mocroft A, Kirk 0, et al. Interruption of combination antiretroviral therapy and risk of clinical disease progression to AIDS or death. HIV Med 2007;8:96-104.

41. Pai NP, Lawrence J, Reingold AL, Tulsky JP. Structured treatment interruptions (STI) in chronic unsuppressed HIV infection in adults. Cochrane Database Syst Rev 2006:Cd006148.

42. Liao CH, Chi CY, Wang YJ, et al. Different presentations and outcomes between HIVinfected and HIV-uninfected patients with Cryptococcal meningitis. J Microbiol Immunol Infect 2012;45:296-304.

43. Boulware DR, Meya DB, Bergemann TL, et al. Clinical features and serum biomarkers in HIV immune reconstitution inflammatory syndrome after cryptococcal meningitis: a prospective cohort study. PLoS Med 2010;7:e1000384.

44. Haddow LJ, Colebunders R, Meintjes G, et al. Cryptococcal immune reconstitution inflammatory syndrome in HIV-1-infected individuals: proposed clinical case definitions. Lancet Infect Dis 2010;10:791-802.

45. Boulware DR, Bonham SC, Meya DB, et al. Paucity of initial cerebrospinal fluid inflammation in cryptococcal meningitis is associated with subsequent immune reconstitution inflammatory syndrome. $\mathrm{J}$ Infect Dis 2010;202:962-70.

46. Mitchell DH, Sorrell TC, Allworth AM, et al. Cryptococcal disease of the CNS in immunocompetent hosts: influence of cryptococcal variety on clinical manifestations and outcome. Clin Infect Dis 1995;20:611-6.

47. Delattin N, Cammue BP, Thevissen K. Reactive oxygen species-inducing antifungal agents and their activity against fungal biofilms. Future Med Chem 2014;6:77-90.

48. Albuquerque P, Nicola AM, Nieves E, et al. Quorum sensing-mediated, cell densitydependent regulation of growth and virulence in Cryptococcus neoformans. MBio 2013;5:e00986-13

49. Bose I, Reese AJ, Ory JJ, et al. A yeast under cover: the capsule of Cryptococcus neoformans. Eukaryot Cell 2003;2:655-63.

50. Cordoba S, Afeltra J, Vitale RG. Evaluation of the in vitro activity of amphotericin B by time-kill curve methodology against large and small capsulate $C$. neoformans isolates. Diagn Microbiol Infect Dis 2011;71: 260-2.

51. Martinez LR, Casadevall A. Susceptibility of Cryptococcus neoformans biofilms to antifungal agents in vitro. Antimicrob Agents Chemother 2006;50:1021-33.

52. Espinel-Ingroff A, Chowdhary A, CuencaEstrella M, et al. Cryptococcus neoformans-Cryptococcus gattii species complex: an international study of wild-type susceptibility endpoint distributions and epidemiological cutoff values for amphotericin B and flucytosine. Antimicrob Agents Chemother 2012;56:3107-13.

53. Schwartz S, Ruhnke M, Ribaud P, et al. Improved outcome in central nervous system aspergillosis, using voriconazole treatment. Blood 2005;106:2641-5.

54. Nau R, Sorgel F, Eiffert H. Penetration of drugs through the blood-cerebrospinal fluid/blood-brain barrier for treatment of central nervous system infections. Clin Microbiol Rev 2010;23:858-83.

55. Yuchong C, Fubin C, Jianghan C, et al. Cryptococcosis in China (1985-2010): review of cases from Chinese database. Mycopathologia 2012;173:329-35.

56. Yao Z, Liao W, Wen H. Antifungal therapy for treatment of cryptococcal meningitis. Chin Med J (Engl) 2000;113:178-80. 
57. Yuchong C, Jianghan C, Hai W, Julin G. Lumbar puncture drainage with intrathecal injection of amphotericin B for control of cryptococcal meningitis. Mycoses 2011;54:e248-51.

58. Legris T, Massad M, Purgus R, et al. Immune reconstitution inflammatory syndrome mimicking relapsing cryptococcal meningitis in a renal transplant recipient. Transpl Infect Dis 2011;13:303-8.

59. Lodha A, Haran M. Is it recurrent cryptococcal meningitis or immune reconstitution inflammatory syndrome? Int J STD AIDS 2009;20:666-7.

60. Horiuchi K, Yamada M, Shirai S, et al. [A case of successful treatment of brain and lung cryptococcosis caused by Cryptococcus gattii]. Rinsho Shinkeigaku 2012;52:166-71. [Article in Japanese].

61. Chen SC, Korman TM, Slavin MA, et al. Antifungal therapy and management of complications of cryptococcosis due to Cryptococcus gattii. Clin Infect Dis 2013;57: 543-51.

62. Ferreira RC, Phan G, Bateman JB. Favorable visual outcome in cryptococcal meningitis. Am J Ophthalmol 1997;124: 558-60.

63. Seaton RA, Verma N, Naraqi S, et al. The effect of corticosteroids on visual loss in Cryptococcus neoformans var. gattii meningitis. Trans R Soc Trop Med Hyg
1997;91:50-2.

64. Rex JH, Larsen RA, Dismukes WE, et al. Catastrophic visual loss due to Cryptococcus neoformans meningitis. Medicine (Baltimore) 1993;72:207-24.

65. Inada T, Imamura $\mathrm{H}$, Kawamoto $\mathrm{M}$, et al. [Cryptococcus Neoformans Var. Gattii meningoencephalitis with cryptococcoma in an immunocompetent patient successfully treated by surgical resection]. No Shinkei Geka 2014;42:123-7. [Article in Japanese].

66. Kanaly CW, Selznick LA, Cummings TJ, Adamson DC. Cerebellar cryptococcoma in a patient with undiagnosed sarcoidosis: case report. Neurosurgery 2007;60:E571. 